\title{
Implications Of The Homomorphism Definition Of Measurement On Accounting Measurement Theory
}

Saratiel Wedzerai Musvoto, North West University - Vaal Triangle Campus, South Africa

\begin{abstract}
This study compares the principles of the representational theory of measurement with accounting practices to decipher the reasons creating a gap between accounting measurement practices and the scientific practices of measurement. Representational measurement establishes measurement in social scientific disciplines such as accounting. The discussion in this study focuses on the need for accounting to provide principled arguments to justify its status as a measurement discipline. The arguments made highlight the need for possible modifications of the accounting measurement concept to deal with issues that are at least partially philosophical in nature, such as the concept of error and the passing of value representations from finite to continuum. These problems are primarily conceptual in nature. They indicate that accounting is far from a measurement discipline. Their resolution could require major changes to the accounting concept of measurement.
\end{abstract}

Keywords: measurement; representational measurement; homomorphism; automorphism

\section{INTRODUCTION}

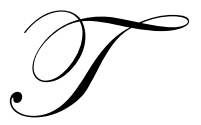

he aim of this study is to discuss a set of problems that preclude accounting from being a true measurement discipline. A review of accounting literature indicates that there is consensus in the literature (see, Ijiri, 1975; Willet, 1987; Chambers, 1997; Staubus, 2004; Musvoto, 2008) that accounting researchers have not succeeded in creating a theory of accounting measurement from accounting practices. However, according to the principles of representational theory of measurement (see, Luce et al, 1971) which establish measurement in social scientific disciplines such as accounting, all processes of measurement must have an underlying theory of measurement. Consequently, it is clear that accounting practices fall short of scientific measurement practices.

The discussion in this study compares the definitions of major accounting concepts with the definitions of the principles of the representational theory of measurement. This comparison discusses a range of probably difficult issues that need clarification. The first issue covered in section 2 concerns an outline of the principles of the representational theory of measurement. It outlines principled arguments that justify the representational theory of measurement and its uses in social sciences. The second issue also dealt with in section 2 covers the history of the development of measurement theory in accounting and its inability to keep pace with the development of the modern principles of measurement. The third type covered in section 3 focuses on issues that are technical and abstract in nature relating to the specific applications of the principles of the representational theory to accounting measurement situations. The measurement issues discussed are primarily conceptual and consequently, they call for either the development of measurement foundations for accounting or the reclassification of accounting as something else other than a measurement discipline. Finally, the conclusion to the study and recommendations to address the problems are covered in section 4. 


\section{ACCOUNTING MEASUREMENT CONCEPT AND THE HISTORICAL BACKGROUND OF REPRESENTATIONAL MEASUREMENT}

The principles of the representational theory of measurement are used in this study to highlight the areas of accounting the discipline has to modify in order to be a true a measurement. Applications of the principles of representation measurement to accounting have been made to investigate if accounting is a true measurement discipline. Authors such as Musvoto (2008), Chambers (1997), Willet $(1987,1988)$ and Orbach (1978) used the five main principles of representational measurement to validate the assertion that accounting is a measurement discipline. These ventures were prompted by the fact that accounting is referred to as a measurement discipline in the absence of evidence to support it. According to Ryan et al (2002) every process of measurement must have an underlying theory of measurement. As a consequence, this pointed towards the view that accounting practices fall short of true practices of measurement. Luce and Narens (1994) summarise the formal part of the principles of the representational theory of measurement and reduced them to five main ideas italicised below that were used by Musvoto (2008) as follows:

1. “A qualitative situation is specified by a (usually ordered) relational structure $X$ consisting of a domain X, of infinitely many relations of $X$ and infinitely many special elements of $X$.

These relations, subsets, and elements are called the attributes of $X$. Measurement axioms are then stated in terms of the attributes of $X$. These axioms are intended to be true statements about $X$ for some empirical identification and are intended to capture important empirical properties of $X$, usually ones that prove useful in constructing measurements of its domain, $X$."

2. "The representational theory requires that the primitives of $\mathrm{X}$ be given an empirical identification.

In particular, if $R$ is an $n$-ary primitive relation on $X$, then it is required that the truth or falsity of $R(x i, \ldots$ $x n$,), for any particular choice of the $n$-tuples xi, be empirically decidable."

3. "As much as possible, measurement axioms, stated in terms of the empirically identified primitives, should be empirically testable"

4. "Measurement of $\mathrm{S}$ is said to take place if and only if the following two theorems can be shown:

$i$. $\quad$ (Existence Theorem). $\mathrm{S}(\mathrm{x})$ is non-empty for each $\mathrm{X}$ that satisfies the measurement axioms.

ii. (Uniqueness Theorem). An explicit description is provided about how the elements of $S(x)$ relate to one another. In practice this description usually consists of specifying a group of functions $\mathrm{G}$ for each 4 in $\mathrm{S}(\mathrm{x})$. $\mathrm{S}(\mathrm{x})=\{\mathrm{g} * \mathrm{~g}: \mathrm{g}$ is in $\mathrm{G})$, where * denotes function composition".

5. "The representational theory of measurement identifies empirical significance with meaningfulness.

The concept of meaningfulness with respect to $S(X)$ is easily extended to numerical statements involving measurements of elements of the domain: meaningful statements are those whose truth-value is unaffected by the particular representation in $S(x)$ used to measure X".

This theory of measurement was developed by Scott and Suppes in 1958. It is a theory of measurement that has as a focal point the viewpoint that all measurements must have a scale that is defined by a set of structure preserving mappings from a qualitative or empirically based structure onto an abstract structure. This means that a particular representation must have empirical properties that are exclusive to it. That is, all measurements must express a clear unambiguous description of the phenomenon they are describing. In addition, statements leading to the formation of scales must be empirically verifiable. This would confirm the existence of the physical substance of the property the measurement purports to be measuring. Furthermore, this assigns unique properties to how the scale assigns a particular property to members of the domain. As noted in the extract, meaning is only achieved if measures are empirically significant. That is to say, the meaning of the underlying empirical relational structure must not be changed by representations used in measuring it. 
The history of the development of the accounting concept of measurement indicates that accountants have misunderstood the concept of measurement and it has lagged behind in evolution to the modern measurement theory. Before the conceptualization of the representational theory by Scott and Suppes (1958), the precise definition of the word "measurement "was lacking. According to Orbach (1978) the definition of measurement arbitrarily depended upon a particular author defining the word. This means that the meaning of measurement varied with the author. For example, Campbell (1952) points that it is necessary the measurement structure be so defined as to satisfy the axioms of additivity. This implies non-additive measurement structures are automatically excluded from this definition. On the other hand Stevens (1951) asserts that measurement can only be achieved if there is a consistent rule of assignment. It is clear from this that random assignment is excluded. It follows that this definition points towards the need for measurements to be empirically verifiable. This is consistent with the view from above, that is, a scale of measurement must be present and measurements must be meaningful.

Caws (1959: 5) defines measurement as the assignment of particular mathematical characteristics to conceptual entities in such a way as to permit an unambiguous mathematical description of every situation involving the entity and the arrangement of all occurrences of it be in a quasi-serial order. This description of measurement reinforces the empirical view of a number. That is, numbers are taken as empirical entities that possess the properties of the entities they are supposed to represent. It is important to note that this view excludes the modern view of nominal measurement. But, it also approves ordinal measurement as it requires the identification of a property that induces a non-metrical order among the situations involving the occurrences of the entity. Nevertheless, the general perspective from this definition is to move towards the modern view of measurement that requires the empirical relational structure and its primitives to be empirically testable. remarked:

Scott and Suppes (1958:116) laid foundations for the modern definition of measurement when they

A primary aim of measurement is to provide a means of convenient computation. Practical control or prediction of empirical phenomena requires that unified, widely applicable methods of analyzing important relationships between the phenomena be developed. Imbedding the discovered relations in various numerical relational systems is the most important such unifying method that has yet been found.

This definition of measurement initiated the move towards the modern principles of measurement outlined above. It advocates the need for measurement to be universal in the community of discussion. Furthermore, the need to have "practical control or prediction of a phenomenon" highlighted requires the development of a theory of measurement. It is also clear that the discovery of relations requires the development of suitable axioms that are intended to be true statements about an empirical phenomenon for some empirical identification. In addition these axioms are intended to capture important empirical properties of the phenomenon usually ones that prove useful in constructing measurements of its domain. The concept of embedding "the discovered relations in various numerical relational systems" introduces the need to discover (homomorphism) functions that map the empirical properties of the phenomenon onto an abstract structure. Embedding discovered relations requires proving the existence and uniqueness theorems. Evidently, this view of measurement is consistent with the modern homomorphism definition of measurement that has come to be widely accepted in social sciences.

Mock (1976:15) also points out that the assignment is representative "if predefined relationships among the assigned numbers represent equivalent relationships that exist among the measured attributes". This also stresses the point that the assignment must be a homomorphism appropriately defined. That is, it must be possible to prove the existence and uniqueness theorems on the relationships among the assigned numbers. The current definition is explained by Narens (2002:760) as follows:

A qualitative structure $\boldsymbol{X}$ is selected to capture the domain $\boldsymbol{A}$ of interest; a mathematical representing structure $\boldsymbol{N}$ is selected to measure $\boldsymbol{X}$ in terms of the scale $\boldsymbol{S}$ of homomorphisms of $\boldsymbol{X}$ into $\boldsymbol{N}$; and meaningfulness is identified with a form of invariance associate $\boldsymbol{S}$, e.g. with $\mathrm{S}$ - invariance.

This definition of measurement is the view of measurement that has come to be known as the representational theory of measurement or the modern principles of measurement. As outlined it advocates the 
specification of the domain of measurement, the measurement function and the value of the measurement function for measurement to occur. Furthermore meaningfulness is identified with the specification of the units of measurement. In conclusion, the discussion above indicates the evolution of the definition of measurement from one that was vague and ambiguous to one that is dichotomous.

However, the same is not true with the accounting discipline. The definition of measurement lagged behind in evolution to the modern principles of measurement. The changes that have occurred in the theory of measurement applicable to social sciences have not found their way to the accounting discipline. For example, before the conceptualization of the representational theory of measurement by Scott and Suppes (1958), the accounting discipline viewed measurement as the assignment of monetary units to the elements of the financial statements at which they can be recognized (see, Gilman, 1939; Paton and Littleton, 1940; Littleton, 1953). As recently as the IASB (2009) pronouncements measurement in accounting is defined as the process of assigning monetary units to the elements of the financial statements amounts at which they can be recognized. The most obvious conclusion to be drawn from this is that the accounting concept of measurement has not changed in tandem with the modern principles of measurement.

In spite of this lack of attention given to measurement by the accounting discipline, some accounting researchers have attempted to introduce the modern principles of measurement to accounting, although without success. For example, Ijiri, (1965) made an attempt to introduce the principles of representational measurement to accounting through the value allocation rule of measurement. The value allocation rule asserts that the adding of values allocated to outgoing resources produces a meaningful value for the decrease in resources resulting from an exchange. This use of the concept of addition in finding the aggregate value of outgoing resources indicates the use of the principles of extensive measurement. The application of extensive measurement to non-physical attributes of objects needs an adequate interpretation for the concatenation operation (Luce et al.1971). But, Musvoto (2008) notes the addition operation in accounting is not given a qualitative interpretation so that the resulting total has an empirical interpretation. Evidently, it is clear that, in the absence of an adequate interpretation of this operation, measurements that are not similar cannot be added to each other.

Willet (1992) expresses that depreciation possesses measurement dimensions that are synonymous with fundamental measurement. He outlines that depreciation is defined as a time average with measurement dimensions of cost per unit of time. This makes depreciation a derived measurement. This means that for depreciation to be a process of measurement, it is necessary to have a theory of value measurement, a theory of monetary units measurement and a theory of time measurement. Furthermore, it is also necessary to have a theory that relates the measures of value, monetary units and time to each other for depreciation measurement to occur. But, Musvoto (2008) notes that there is a ratio scale measurement for monetary units and time intervals. But no comparable structure exits for the measurement of value. In addition, there is no theory of measurement that connects the measures of monetary units, value and for time, which is a necessity for derived measurement. It follows that there is no empirical relation between, value, the concatenations that pertain to time measurement and the concatenations that pertain to monetary unit measurement. Therefore, it can be concluded that depreciation is not a fundamental measurement, as it has no natural theory of measurement that supports it. This demonstrates a lack of understanding of the principles of representational measurement.

\section{ACCOUNTING MEASUREMENT IN THE REPRESENTATIONAL MEASUREMENT CONTEXT}

The criticisms of the concept of accounting measurement based on the homomorphism definition of measurement are of two types: those that pertain to the arguments advocating that the definition of accounting measurement is too narrow in the sense that it does not account for certain kinds of numerical assignments apart from those that involve the assignment of monetary units to the elements of the financial statements; and those arguing that the concept of accounting measurement is too broad in that it permits too many kinds of monetary assignments to the elements of the financial statements. The former is usually brought about by the fact that it seems that in characterizing accounting measurement as the assignment of monetary units to the elements of the financial statements, the proponents of the accounting measurement theory have fastened onto something which is undoubtedly of great importance in modern accounting. However, this characterization of accounting measurement is by no means an essential feature of measurement. What is important in measurement is that the transformation of 
an empirical relational structure onto a numerical relational structure be through a homomorphism (Narens, 2002). There is no good reason given, for example, by IASB (2009), about why what is considered accounting measurement should be restricted to the assignment of monetary units to the elements of the financial statements. The only important thing in accounting measurement is that the information in the financial statements provides a sophisticated and convenient conceptual framework that can be used in describing the financial activities of an entity. As a result it seems absurd to say that other concepts of measurement do not measure in accounting because they do not assign monetary units to the elements of the financial statements. The employment of abstract structures other than monetary units in other disciplines in describing an empirical relational structure is not essentially different from the employment of monetary units as an abstract structure that represents the value of an element of the financial statements. It follows from this that the employment of a particular abstract structure (monetary units) to represent a particular empirical relational structure cannot be used as a definition of measurement. As a consequence, this severely limits the description of measurements in a discipline to describing the representation of a particular empirical relational structure with a particular abstract structure.

Musvoto (2008) criticizes the accounting definition of measurement as being too broad. He notes that requiring that value be represented by monetary units in the absence of a specified set of homomorphisms that map the empirical structure of value onto monetary unit places little restriction on the uniqueness of representations. Furthermore the inadequate description of the structure of value (see, Ryan et al, 2002) also implies that requiring that the value of an element of the financial statements be representable into the structure of monetary units and real numbers places little restriction on the structure beyond monetary units and cardinality. This viewpoint of measurement does not involve any concept of representational measurement. Indeed the principles of the representational theory of measurement would hardly be prepared to accept the accounting definition of measurement as a sufficient criterion for a discipline to be called a measurement discipline. It is seems that what is lacking here is an analysis of what it should mean that the value of an element of the financial statements is representable by monetary units.

The above criticisms of the concept of accounting measurement suggest the following problem:

Problem 1 - Justify the concept of accounting measurement as a general theory of accounting measurement in a philosophically principled manner without restricting its uses to the assignment of monetary units to the elements of the financial statements

Theories of value measurement in accounting are alluded to by the IASB (2009), although based on the quantification of monetary units are not in principle ruled out by the principles of the representational theory of measurement. According to Luce and Narens (1994) empirical structures can be based on numerical information just as long as those numbers arise from empirical means. In accounting this is the case; the numerosity of monetary units is assigned to reflect the value of an element of the financial statements by concatenating the monetary units so that they can be represented by positive real numbers. What is lacking from the perspective of representational measurement, are the axiomatic theories for the various kinds of value measurement. For example, Ryan et al (2002) points out that there is no agreement relating monetary units to the value of an element of the financial statements. This indicates there is no specified function that maps value onto the structure of monetary units. In the accounting discipline there are no impediments in principle to the development of axiomatic theories of value measurement, although in practice over the past centuries none has been created and moreover, it appears to be a difficult task.

\subsection{Error In Accounting Measurement Theory}

This section deals with the criticisms of accounting measurement that concern how error should be treated in relation to measurement models. In accounting measurement, probabilities underlie the recognition of the elements of the financial statements. For example, the IASB (2009) points out that the concept of probability is used in the recognition criteria to refer to the degree of uncertainty that the future economic benefits associated with the item will flow to or from the entity. The introduction of the concept of probability creates three problems concerning the treatment of error that are intimately connected with the concept of accounting measurement. 
According to Orbach (1978) expectations have legitimate properties in the present that are measurable. This means that probabilities underlie the observations that are made. The first of these problems is based on the viewpoint that there is currently no mechanism in the accounting discipline to test the measurement axiomatizations that underlie probabilities. This is exacerbated by the fact that the representational measurement theory has not made much progress in devising a system that tests the measurement aximatizations underlying probabilities (Falmagne, 1979, 1980; Michell, 1986). These criticisms of the concept of accounting measurement suggest the following problem:

Problem 2 - The accounting literature must specify a probabilistic version of accounting measurement theory and the related statistical techniques for evaluating whether or not a data set supports or refutes certain accounting measurement axioms

The most obvious approach taken is to assume that probabilities are representation of a corresponding existing underlying random variable in which the largest value observed determines the choice. Since, the accounting literature (IASB, 2009) views the procedure of assigning monetary units to the elements of the financial statements as leading to fundamental measurements of value, this approach is not satisfactory because it assumes as primitive the numerical structure of probabilities and thus places the description of randomness at a numerical, rather than qualitative level. In representational measurement the qualitative primitives have to be formulated so as to simultaneously capture both the structural and the random qualities of the situation. For example, in the accounting literature, a typical extensive situation is assumed where both judgments of order are made and entities are concatenated to form new ones believed to be exhibiting the same attribute (value). For instance, under current assets in the statement of financial position, the expected values of individual current assets (e.g., inventory and debtors) are added to form a new entity; total current assets which, exhibits the same attribute (value). It would be necessary to have axioms that lead to a random variable representation that specifies the random variable with respect both to their structural relations with each other and to the nature of their distributions. That is to say, in the accounting discipline addition denotes the concatenation within the extensive structure (monetary units), as a result it is necessary to arrive at qualitative axioms that guarantee that monetary units can be taken to represent the value of total current assets. The problem is to find an equation that guarantees that the expected values of individual current assets can be added to give total current assets. Currently, there is none given in the accounting discipline. In this case, it would be expected to find in the accounting literature the distribution of the random variable associated to the addition of individual current assets (total current assets) to be the convolution of the distributions of those associated to individual current assets. This would conform to the traditional measurement representation, that is: $\mathrm{X}$ $+\mathrm{Y}=\mathrm{Z}$ iff $\mathrm{E}\{\mathrm{f}(\mathrm{x})\}+\mathrm{E}\{\mathrm{f}(\mathrm{y})\}=\mathrm{E}\{\mathrm{f}(\mathrm{z})\}$. This discussion creates the following problem:

Problem 3 - Extend the qualitative primitives of accounting measurement theory in such a way that the objects of the domain are represented by random variables instead of by numbers.

It is generally agreed in the accounting literature (see, Tinker, 1985), Musvoto, 2008; Ryan et al 2002, Stamp, 1981) that value is inherently a property in flux. But, according to Luce \& Narens (1994) numbers are constant random variables. Given this perspective, the borne of contention is how it should be determined whether the variability of the observed values of the property (value) is intrinsic to it or based on the fact that the accounting literature's description of it is incomplete. It could be that the cause of value to be a property in flux is conditioned by other properties that the accounting literature has not taken into account when measuring it. It follows that the difficulty in developing a theory of measurement in accounting may be related not to an absence of measurable qualities of value, but to the fact that these qualities are involved with many others such that an isolation of a property to measure is not possible. Consequently, if measurement is to play a crucial role in accounting more work is necessary in this respect. If the qualitative structure of value is not apparent, it may be necessary to measure value in contexts that could be analogous to extensive and conjoint measurement, but not preference among uncertain alternative as this would place randomness on a numerical rather than qualitative level. It is important to note that the viewpoint that randomness can be captured quantitatively is not regarded as false in this discussion. Theories of subjective utility theory (Savage, 1954) have done just that. In these theories, a random variable (utility) and probability distributions over events underlying the uncertain alternatives is inferred from choices among uncertain alternatives. Again as with the determination of the qualitative structure of value, utility does not have an apparent empirical relational structure, but it is affected by many other qualities that are difficult to isolate. This points 
towards the potential use of multi-valued logics and fuzzy logic theories that are useful in dealing with problems that involve ambiguous states of nature. Consequently, this discussion creates the following problem for accounting measurement:

Problem 4 - Extend the accounting theory of measurement to include axioms formulated in terms of multi-valued logics

\subsection{The Concept Of Meaningfulness In Accounting Measurement}

The concept of meaningfulness has not received much attention in the accounting discipline as the need to assign monetary units to the value of the items to achieve recognition as a result it is less developed. In particular the criterion of meaningfulness invoked by the accounting literature has not been fully justified. For example, the IASB (2009) points out that accounting information must be prepared in way that makes it useful to the users. In representational measurement (Luce et al, 1990) measurement information is useful if it is meaningful. It follows that if accounting is a measurement discipline as outlined in the literature (IASB, 2009), then it means accounting information as a product of a social scientific discipline must comply with the representational measurement concept of meaningfulness. As noted in section 2 the representational theory of measurement equates meaningfulness with empirical significance. This means that the qualitative correlates describing the relations of the empirical structure of value should be definable empirically in terms of the primitives of the empirical structure of value if accounting information is to be considered meaningful. This also points to the fact that correlates of non-meaningful qualitative relations of the empirical relational structure of value are non-empirical with respect to the primitives. However, authors such as Ryan et al (2002), Stamp (1981) and Musvoto (2008) point out that the qualitative structure of value is vague and ambiguous and as result the relationship between value and monetary units cannot be specified. This means that measures of value are not empirically significant. In other words value measures are not invariant. This suggests that the meaningfulness of accounting information cannot be determined by equating it with empirical significance. But, if accounting information is supposed to be useful to users this suggests that accounting information must have a pragmatic value in the context of users. If accounting is a measurement discipline as outlined in the literature (IASB, 2009), then it should be clear to the users the use to which the accounting information can be put. This discussion leads to the following problem:

Problem 5 - The accounting measurement theory of meaningfulness should be amended so that it can equate meaningfulness with empirical significance in order to be consistent with representational measurement otherwise, it must outline conditions of meaningfulness for accounting information.

\subsection{Accounting And Continuum Representations}

The definition of accounting measurement as the assignment of monetary units to represent the value of an element of the financial characterizes the structure of value as continuous. It defines the structure of value as an infinite standard sequence. It follows that by assigning monetary units to represent the value of an element of the financial statements, in essence, one axiomatizes a finite standard sequence which leads to an integer representation and a uniqueness theorem. However, if value is taken to be relative to an individual it leads to a set of inequalities that must be satisfied, which establishes the existence of a numerical representation, but one is unable to give a compact and useful characterization of its uniqueness. It is represented by a range of values that covers all the positive integers. Consequently, the assignment of monetary units to represent the values of the elements of the financial statements becomes a stochastic process whose outcome is contained in the set of positive integers. It follows that, the question that needs to be answered is why does, the accounting discipline, have only to look at the finite structure of value neglecting its continuum structure. It is possible to conceive some sort of accounting measurement theory containing finite structures of value that leads to a continuous structure from which the finite structures can be thought of as samples. Therefore, this discussion leads to the following problem:

Problem 6 - Provide a principled account of why in an accounting measurement theory it is scientifically useful to replace continuum structures by finite ones 


\subsection{The Compatibility Of Psychological And Physical Theory In Accounting}

The assignment of monetary units to the value of an element of the financial statements requires that the agent matches the attribute of value in the empirical relational structure of value with the attribute of money in the empirical relational structure of money. Formally, there should be two physical structures ( $\mathbf{V}$ ' for value and $\mathbf{M}$ ' for monetary units) with domains $\mathbf{V}$ and $\mathbf{M}$; given, that monetary units as the representing structure are an extensive structure, then, each of these physical structures should have a ratio scale representation. In this case the psychological information may be given as follows: for each attribute $\mathbf{v}$ in domain $\mathbf{V}$ there is some $\mathbf{m}$ in domain $\mathbf{M}$ such that according to the agent $\mathbf{m}$ matches v. Since, Musvoto (2008), Ryan et al (2002) points out that there is no specified relation that relates value to monetary units it means that there are two possible physical relational structures that are connected by a purely psychological relation. As a result, if both value and monetary units are taken to be structures that are based on the ratio scale as implied in the accounting literature, an approximation of the matching relation between these two structures can be described as a power relation. However, Musvoto (2008) also asserts that value is a hypothetical phenomenon that has not received full measurement analysis. This suggests that there is one physical relational structure connected to a hypothetical phenomenon by a psychological relation. Thus, this requires one to find the correspondent of the power relation. Luce and Narens (1994:239) outline a principle of compatibility between two physical extensive structures as follows:

To each translation $t$ of $X$, there is a corresponding translation $r t$ of $S$ such that for all $x$ that is in $X$ and for all s that is $S: x M s$ if and only if $t(x) M r t(s)$.

This shows that two physical structures have compatible domains if the translation of an attribute in one domain matches the translation of an attribute in another domain. In this case the relation $\mathrm{M}$ connecting the two structures is purely psychological. According to Luce and Narens (1994) the matching relation can be described empirically as a power relation (at least to a first approximation) between the two physical ratio-scale measures. This means that the dimensions of the multiplicative constant of the relation depend upon the exponent used. Furthermore it is clear that the application of the principle of compatibility between two domains is highly special to a specific situation. It follows that in the accounting discipline it is necessary to specify the domain of value and the domain of money before matching can occur between the members of these two domains. If value is a hypothetical phenomenon as outlined above, then it means that the compatibility between the domain of value and monetary units cannot currently be described as value is not a physical structure. Moreover, although it is clear that the compatibility of the domain of value with the domain of monetary units is unique to a specific situation, the idea of asking which psychological laws are compatible with the translation of the members of domain of value and the members of the domain of monetary units is a general principle in accounting.

Problem 7 - Provide scientific or philosophical grounding for the supposition that a hypothetical phenomenon such as value that is governed by psychological laws should be compatible with the automorphism structures of monetary units. Furthermore, it is necessary to provide examples which illustrate their compatibility or where it is clearly violated.

\section{CONCLUSIONS}

The discussion above highlights that despite positive contributions of accounting measurement theory, it has been subjected to a lot of criticisms from the representational measurement perspective. One basic issue is its failure to give a principled justification for limiting the definition of measurement in accounting to the assignment of monetary units to the value of the elements of the financial statements. This neglects other forms of measurement that may occur in accounting. A further point of interest is that accounting measurement theory neglects structures that do not map into the real number system. It is possible that the structure of value could be mapped onto families of random variables or into structures of multi-valued logics. Furthermore, there is no extension of the qualitative primitives of accounting measurement theory in such a way that the objects of the domain are represented by random variables instead of by constant random variables (numbers).

Value is considered to be inherently a property in flux. This means that it must be valued using multivalued logics. However, in accounting it is measured by mono- valued logics. It follows that the accounting theory 
of measurement does not include axioms formulated in terms of multi-valued logics, when it is possible that the variability of the observed values of the property (value) could be conditioned by other properties that the accounting discipline has not taken into account when measuring it.

Currently, the accounting measurement theory of meaningfulness does not equate meaningfulness with empirical significance as is required by representational measurement. It has to do so in order to be consistent with representational measurement, otherwise, it must outline conditions of meaningfulness for accounting information.

It has been argued that value is a subjective concept that can assume any value in the domain of monetary units. However, without an appropriate justification the accounting discipline uses finite structures to represent the value of a commodity. Consequently, it is necessary to justify why in an accounting measurement theory it is scientifically useful to replace continuum structures by finite ones. Moreover, the accounting discipline approves the matching members of the empirical structure of value to members of the empirical structure of money. But, there is no scientific or philosophical grounding provided for the supposition that a hypothetical phenomenon such as value that is governed by psychological laws should be compatible with the automorphism structures of monetary units. Therefore, it is necessary to provide examples to illustrate such compatibility.

\section{AUTHOR INFORMATION}

Dr. S Wedzerai Musvoto is a senior lecturer in Financial Management and Accounting at the School of Accounting Sciences of the North-West University's Vaal Triangle Campus. He received his PhD from the University of Pretoria in 2008. Dr Musvoto is also affiliated with the Association of Chartered Certified Accountants and the Chartered Institute of Management Accountants. His research interests include accounting measurement, risk management and the implications of modern principles of measurement on finance theory development.

\section{BIBLIOGRAPHY}

1. Campbell, N.R. (1952). What is science? Dover publications, Inc

2. Caws, P. (1959), Definition and Measurement in physics, in C. West Churchman and Philburn Ratoosh, eds. Measurement, Definitions, and theories, John Wiley \& Sons, Inc.

3. Chambers, R.J. (1997) "Wanted: Foundations of Accounting Measurement", Abacus, 34(1): 36-47.

4. $\quad$ Falmagne, J.C., (1980) "A Probabilistic Theory of Extensive Measurement, Philosophy of Science, 47, 277296.

5. Falmagne, J.C., (1979) "On a Class of Probabilistic Conjoint Measurement Models: Some Diagnostic Properties', Journal of Mathematical psychology, 19, 73-88

6. $\quad$ Gilman, S. (1939). Accounting Concepts of Profit, Ronald Press

7. Ijiri, Y. (1965) "Axioms and Structures of Conventional Accounting Measurement", The Accounting Review, 36-53

8. Ijiri, Y. (1975) "Theory of Accounting Measurement”, American Accounting Association

9. International Accounting Standards Board (IASB), 2009, International Financial Reporting Standards (IFRS) (2009): Including International Accounting Standards (IAS) and interpretations as at 1 July 2009.

10. Littleton, A.C. (1953). Structure of Accounting Theory, American Accounting Association

11. Luce, R, D. and Narens, L. (1994) "Fifteen Problems Concerning The Representational Theory of Measurement". Available, http:// www. Imbs.uci.edu / personnel / Luce / 1994/Luce \& Narens_Book \% 20 Chapter 1994.pdf, Accessed 2006/10/10

12. Luce, R. D., Krantz, D.H., Suppes, P. \& Tversk, A. (1971) "Foundations of Measurement, (Vol. 1) Additive and Polynomial representations", New York: Academic Press

13. Michell, J., (1986) "Measurement Scale and Statistics: A clash of Paradigms', Psychological Bulletin, 100, 398-407

14. Mock, T.J. (1976) Measurement and Accounting Information Criteria, Studies in Accounting Research No 13, American Accounting Association

15. Musvoto, S.W (2008) “Applying the representational theory of measurement to accounting”, PhD thesis, University of Pretoria, Pretoria, Viewed yymmdd<http://upetd.up.ac.za/thesis/available/etd-03282009$\underline{125051 />}$ 
16. Narens, L. (2002) “A meaningful justification for the representational Theory of Measurement”, Journal of Mathematical Psychology, 46: 746-768

17. Orbach, K.N. (1978). Accounting as a Mathematical Measurement Theoretic Discipline, Unpublished PhD Thesis, Texas A\& M University

18. Paton, W. A. and Littleton, A.C. (1940) An introduction to Corporate Accounting Standards, American Accounting Association

19. Ryan, B., Scapens, R.W \& Theobald, M. (2002) "Research Method and Methodology in finance and Accounting”, Second Edition, Mitcham, Surrey, International, Padstow, Cornwall

20. $\quad$ Savage, L.J., (1954) "The Foundations of Statistics, New York: Wiley

21. Stamp, E. (1981), "Why can Accounting not become a Science like Physics?", Abacus, June, 13-27

22. Scott, D. \& Suppes, P. (1958) "Foundational Aspects of Theories of Measurement", Journal of Symbolic Logic, 23, 113-128

23. Staubus, G. J. (2004) "Two views of Accounting Measurement”, Abacus, 40 (3): 265-279

24. Tinker, T. (1985) "Paper Prophets, A Social Critique of Accounting". New York: Praeger

25. Willet, R. J. (1987) “An Axiomatic Theory of Accounting measurement”, Accounting and Business Research: 155-171.

26. Willet, R. J. (1988) “An Axiomatic Theory of Accounting Measurement - Part 2", Accounting and Business Research, 19(73): 79-91

27. Willet, R. J. (1992), "Transactions Theory, Stochastic Processes and Derived Accounting measurement", Abacus, Vol. 27, No 2 